\title{
Effects of rare sugar D-allulose on acid production and probiotic activities of dairy lactic acid bacteria
}

\author{
H. Kimoto-Nira, ${ }^{* 1}$ N. Moriya, ${ }^{*}$ S. Hayakawa,† K. Kuramasu,‡ H. Ohmori, ${ }^{*}$ S. Yamasaki,§ and M. Ogawa† \\ *NARO Institute of Livestock and Grassland Science, 2 Ikenodai, Tsukuba, Ibaraki 305-0901, Japan \\ †Kagawa University Faculty of Agriculture, 2393 Ikenobe, Mikicho, Kita, Kagawa, 761-0795, Japan \\ łKagawa University Center for Social Collaboration and Intellectual Property, 2217-20 Hayashicho, Takamatsu, Kagawa, 761-0396, Japan \\ §Japan International Research Center for Agricultural Sciences, Ohwashi 1-1, Tsukuba, Ibaraki 305-8686, Japan
}

\begin{abstract}
It has recently been reported that the rare sugar Dallulose has beneficial effects, including the suppression of postprandial blood glucose elevation in humans, and can be substituted for sucrose as a low-calorie food ingredient. To examine the applications of D-allulose in the dairy industry, we investigated the effects of Dallulose on the acid production of 8 strains of yogurt starter (Lactobacillus delbrueckii ssp. bulgaricus and Streptococcus thermophilus) and 4 strains of lactococci, including potential probiotic candidates derived from dairy products. Acid production by 2 L. delbrueckii ssp. bulgaricus yogurt starter strains in milk was suppressed by D-allulose, but this phenomenon was also observed in some strains with another sugar (xylose), a sugar alcohol (sorbitol), or both. In contrast, among the dairy probiotic candidates, Lactococcus lactis $\mathrm{H} 61$, which has beneficial effects for human skin when drunk as part of fermented milk, was the only strain that showed suppression of acid production in the presence of D-allulose. Strain H61 did not metabolize D-allulose. We did not observe suppression of acid production by strain H61 with the addition of xylose or sorbitol, and xylose and sorbitol were not metabolized by strain H61. The acid production of strain H61 after culture in a constituted medium (tryptone-yeast extract-glucose broth) was also suppressed with the addition of D-allulose, but growth efficiency and sugar fermentation style were not altered. Probiotic activities - such as the angiotensinconverting enzyme inhibitory activity of H61-fermented milk and the superoxide dismutase activity of H61 cells grown in tryptone-yeast extract-glucose broth-were not affected by D-allulose. D-Allulose may suppress acid production in certain lactic acid bacteria without altering their probiotic activity. It may be useful for
\end{abstract}

Received October 26, 2016.

Accepted March 8, 2017.

${ }^{1}$ Corresponding author: anne@affrc.go.jp developing new probiotic dairy products from probiotic strains such as Lactococcus lactis $\mathrm{H} 61$.

Key words: rare sugar, D-allulose, fermented milk, lactic acid bacteria

\section{INTRODUCTION}

The rare sugar D-allulose is a C-3 epimer of D-fructose. Such monosaccharides and their derivatives exist in relatively small amounts in nature. Because of the low abundance of this sugar, its biological functions have not been thoroughly evaluated. However, an innovative method has been developed for the mass production of D-allulose by the continuous epimerization of D-fructose using immobilized D-tagatose 3-epimerase (Takeshita et al., 2000), and this method has contributed to the progress of research into rare sugars. Toxicity tests have shown that D-allulose is safe as a food material. For example, intake of $5 \mathrm{~g}$ of D-allulose with meals 3 times a day for 12 continuous weeks in 17 human subjects yielded neither abnormal effects nor clinical problems (Hayashi et al., 2010). In addition, D-allulose was given Generally Recognized as Safe status by the US Food and Drug Administration in August 2011 (GRN No. 400). D-Allulose has $70 \%$ of the sweetness of sucrose and is low in calories, at $0.39 \mathrm{kcal} / \mathrm{g}$. For these reasons, interest is growing in its use as a sweetener in place of sucrose or high-fructose corn syrup in the food industry (Mu et al., 2012).

Many studies of the beneficial effects of D-allulose on human health are already available. Iida et al. (2008) reported that D-allulose was effective in suppressing blood glucose elevations after eating in humans. Hayashi et al. (2010) also reported that intake of D-allulose suppressed postprandial blood glucose elevation in human subjects with borderline diabetes. Inhibition of the absorption of D-glucose from the intestine (Hishiike et al., 2013) are possible mechanisms for this effect. D-Allulose has also been shown to inhibit hepatic fatty acid synthase by controlling adipose tissue deposition; 
compared with D-glucose, it caused less body weight gain in rats (Matsuo et al., 2001).

Considerable research has focused on the beneficial effects for human health of yogurts made with Lactobacillus delbrueckii ssp. bulgaricus or Streptococcus thermophilus and fermented milks made from other lactic acid bacteria or bifidobacteria. For example, casein hydrolysate containing Val-Pro-Pro and Ile-Pro-Pro, which inhibits angiotensin-converting enzyme (ACE; Donkor et al., 2007), has been found to ameliorate vascular endothelial dysfunction in human subjects with mild hypertension (Hirota et al., 2007) and improve both central systolic blood pressure and the brachialankle pulse, a marker of arterial stiffness (Nakamura et al., 2011).

Superoxide dismutase (SOD, EC 1.15.1.1) enzymes, which catalyze dismutation of the superoxide radical $\left(2 \mathrm{O}_{2}{ }^{\bullet-}+2 \mathrm{H}^{+} \rightarrow \mathrm{H}_{2} \mathrm{O}_{2}+\mathrm{O}_{2}\right)$, are possible candidates among the compounds in lactic acid bacteria that may have health benefits in humans. Han et al. (2006) found that supplying bacteria that contained a vector carrying the SOD gene to the rat colon improved experimental colitis, suggesting that this strategy offered the promise of clinical application. LeBlanc et al. (2011) reported that in a murine model of trinitrobenzenesulfonic acid-induced Crohn's disease, mice that received SOD-producing lactic acid bacteria recovered faster from weight loss than mice that did not receive the bacteria.

In many countries, consumers have begun to favor many types of organoleptic properties and textures of food (Thompson et al., 2007). If the rare sugar D-allulose is to be used in the dairy industry to develop new products, its effects on the acid production of dairy lactic acid bacteria, including $L$. delbrueckii ssp. bulgaricus and $S$. thermophilus as yogurt starters, should be investigated. In the dairy industry, acid production is one of the important properties of a starter culture (Hébert et al., 2000). In this study, we investigated these effects, and we also analyzed the effects of D-allulose on the growth and probiotic activities (including ACE-inhibitory and antioxidative activities) of several potential probiotic candidates derived from dairy products.

\section{MATERIALS AND METHODS}

\section{Strains and Culture Conditions}

We used 12 strains of dairy lactic acid bacteria (Table 1). With the exception of Lactococcus lactis ssp. cremoris ATCC $19257^{\mathrm{T}}$ (American Type Culture Collection, Manassas, VA), the strains of lactococci had been found in our previous studies to be potential probiotic candidates. Oral administration of strain N7 decreases total serum cholesterol and triglyceride contents in rats (Lee et al., 2005). Strain H61 suppresses hydration loss caused by seasonal change in humans (Kimoto-Nira et al., 2012b). Strain 7-1 has adhesive properties, such as adherence to porcine gastric mucin in vitro (KimotoNira et al., 2015). We used non-probiotic strain ATCC $19257^{\mathrm{T}}$ in this study as a reference strain because it is a type strain of L. lactis ssp. cremoris.

The strains were maintained by subculture of $1 \%$ inocula in de Man, Rogosa, and Sharpe (MRS) broth (Becton Dickinson, Sparks, MD). Cultures were incubated for 18 to $24 \mathrm{~h}$ at $30^{\circ} \mathrm{C}$ (for lactococci) or $37^{\circ} \mathrm{C}$ (for lactobacilli and streptococci). The cultures were stored at $4^{\circ} \mathrm{C}$ between transfers and subcultured once before use.

\section{Effects of D-Allulose on Acid Production in Milk}

Three milliliters of $10 \%$ (wt/vol) skim milk solution was dispensed into each test tube and sterilized by au-

Table 1. Strains of dairy lactic acid bacteria examined

\begin{tabular}{llll}
\hline Bacterium & Strain no. & Origin & Source $^{1}$ \\
\hline Lactobacillus delbrueckii ssp. bulgaricus & $2-5$ & Fermented milk & Laboratory collection \\
& $5-10$ & Fermented milk & Laboratory collection \\
NITE & NITE \\
Streptococcus thermophilus & $6-8$ & Fermented milk & MAFF \\
& $8-1$ & Alcoholic drink from mare milk & Laboratory collection \\
Lactococcus lactis ssp. cremoris & $\mathrm{B} 5-\mathrm{b}$ & Unknown & MAFF \\
& $2-8$ & Fermented milk & MAFF \\
Lactococcus lactis ssp. lactis & 501 & Unknown & MAFF \\
NITE & Commercial cheese starter & ATCC \\
\hline
\end{tabular}

${ }^{1}$ ATCC $=$ American Type Culture Collection (Manassas, VA); MAFF = Ministry of Agriculture, Forestry, and Fisheries (Tsukuba, Japan); NITE $=$ National Institute of Technology and Evaluation (Kisarazu, Japan). 
toclaving it at $110^{\circ} \mathrm{C}$ for $10 \mathrm{~min}$. D-Allulose, other sugars (fructose and xylose), and a sugar alcohol (sorbitol) were each dissolved in distilled water to a concentration of $10 \%$ (wt/vol) and then sterilized by passage through a filter $(0.2 \mu \mathrm{m}$; Advantec, Tokyo, Japan). We prepared each medium by adding one of these carbohydrates to the skim milk solution to a final concentration of $0.5 \%$ ( $\mathrm{vol} / \mathrm{vol}$ ), which is used as the sugar concentration suitable for screening a dairy starter (Kimoto-Nira et al., 2012a). Overnight fresh MRS cultures at 30 or $37^{\circ} \mathrm{C}$ were harvested by centrifugation for $15 \mathrm{~min}$ at $1,800 \times$ $g$ at $4^{\circ} \mathrm{C}$; the pellets were washed with sterilized $0.85 \%$ $\mathrm{NaCl}$ solution and then suspended in the same solution. Each medium was inoculated with a $1 \%$ ( $\mathrm{vol} / \mathrm{vol}$ ) bacterial suspension of the test strains and incubated at 30 or $37^{\circ} \mathrm{C}$ for 1 or $2 \mathrm{~d}$ under batch conditions. The $\mathrm{pH}$ value of the culture was then determined.

To test mixtures of 2 yogurt starters, a test tube containing $3 \mathrm{~mL}$ of $10 \%$ skim milk solution was inoculated with $1 \%$ of 2 different strains ( $L$. delbrueckii ssp. bulgaricus and $S$. thermophilus) in mixed culture: the concentration of the inoculum in the mixed culture was therefore $0.5 \%$ for each strain.

\section{Effects of D-Allulose on Bacterial Growth and Acid Production in Constituted Medium}

The effects of D-allulose on bacterial growth and acid production were examined in a medium constituted from $4 \mathrm{~mL}$ of tryptone-yeast extract-glucose (TYG) broth, which is made up of $0.5 \%$ tryptone (Becton Dickinson), $0.5 \%$ yeast extract (Wako Pure Chemical Industries, Tokyo, Japan), 1\% sodium succinate, $1 \%$ sodium chloride, and $1 \%$ glucose. The broth was adjusted to a $\mathrm{pH}$ of approximately 6.3. The broth was inoculated with a $1 \%$ (vol/vol) bacterial suspension of the test strain and incubated at the appropriate growth temperature for 1 to $2 \mathrm{~d}$ under batch conditions.

At the end of the incubation, the molar growth yield on glucose $\left(\mathbf{Y}_{\mathrm{G}}\right)$ was calculated by dividing the dry weight of cells by the molar mass of consumed glucose, as determined below. To measure dry weight, cells were collected by centrifugation $\left(1,800 \times g, 20 \mathrm{~min}, 4^{\circ} \mathrm{C}\right)$, washed once with distilled water, dried at $105^{\circ} \mathrm{C}$ for 4 $\mathrm{h}$, and weighed.

\section{Viable Cell Count}

Viable cells were counted by plating culture samples of the appropriate dilution in $0.85 \% \mathrm{NaCl}$ solution on an MRS plate supplemented with agar (1.6\%). All plates were then incubated at the appropriate growth temperature for $2 \mathrm{~d}$.

\section{Substrate and Metabolite Analysis}

Each of the TYG culture supernatants was filtered through a disposable membrane filter unit (pore size, $0.45 \mu \mathrm{m}$ ) and then stored at $-20^{\circ} \mathrm{C}$ until analysis for substrate and product. Residual glucose in the broth was measured enzymatically with a Glucose CII test kit (Wako Pure Chemical Industries). Allulose was quantified with an HPLC system (Shimadzu, Kyoto, Japan) equipped with a Hitachi Gel pack GL-C611 column (Hitachi Chemical Co. Ltd., Tokyo, Japan) and a RID-10A refractive index detector (Shimadzu). Before HPLC analysis, the D-allulose solution in the broth was treated for $30 \mathrm{~min}$ with a strongly acidic cation-exchange resin (DIAION SK1B; Mitsubishi Chemical Co. Ltd., Tokyo, Japan) and a strongly basic anion-exchange resin (Amberlite IRA411; Organo Co. Ltd., Tokyo, Japan). The pretreated sugar solution was filtered through a disposable membrane filter unit with a pore size of $0.2 \mu \mathrm{m}$. The sugar solution was then injected into a Hitachi Gel pack GL-C611 column heated at $60^{\circ} \mathrm{C}$ and eluted at a flow rate of $1.0 \mathrm{~mL} / \mathrm{min}$ using an isocratic mobile phase of $0.1 \mathrm{~m} M \mathrm{NaOH}$ aquatic solution. The D-allulose concentration was determined by referring to a calibration curve of D-allulose standards.

Lactate, acetate, and formate in the TYG broth after culture were quantified using a post-column HPLC system (LC-UV; Shimadzu) equipped with a Shim-pack SCR-102H column $(8 \times 300 \mathrm{~mm}$; Shimadzu $)$ fitted with a SCR-102H guard column and a CDD-10Avp conductivity detector. The mobile phase contained $5 \mathrm{mM}$ toluenesulfonic acid. The post-column buffer solution was composed of $5 \mathrm{mM} p$-toluenesulfonic acid, $100 \mu M$ EDTA, and $20 \mathrm{~m} M$ Bis-Tris. Ethanol was quantified with an F-kit (Boehringer, Mannheim, Germany).

\section{ACE-Inhibitory Activity}

To measure ACE-inhibitory activity in fermented milk, whey was prepared by centrifuging milk incubated with bacteria at $15,300 \mathrm{~g}$ for $10 \mathrm{~min}$. The $\mathrm{pH}$ of the whey fraction was adjusted to 5.0 with $3 \mathrm{~N} \mathrm{NaOH}$. The buffered solution was diluted 5-fold with water and used to determine enzyme activity with an ACE assay kit (Dojindo Laboratories, Kumamoto, Japan) in accordance with the manufacturer's instructions (https:// www.dojindo.com/TechnicalManual/Manual_A502. pdf). Then, ACE-inhibitory activity was calculated as follows:

$$
\begin{gathered}
\text { ACE-inhibitory activity }(\%)= \\
\left(\mathrm{A}_{\text {blank1 }}-\mathrm{A}_{\text {sample }} / \mathrm{A}_{\text {blank1 }}-\mathrm{A}_{\text {blank2 }}\right) \times 100,
\end{gathered}
$$


Table 2. $\mathrm{pH}$ values after fermentation of milk with or without D-allulose, ${ }^{1}$ to which pure cultures of yogurt starters were added

\begin{tabular}{lcc}
\hline & \multicolumn{2}{c}{$\mathrm{pH}^{2}$} \\
\cline { 2 - 3 } Strain & Control & Allulose \\
\hline Lactobacillus delbrueckii ssp. bulgaricus & $3.73 \pm 0.01$ & $3.75 \pm 0.01$ \\
$2-5$ & $4.03 \pm 0.01$ & $4.05 \pm 0.01$ \\
$5-10$ & $3.94 \pm 0.01$ & $4.15 \pm 0.01^{*}$ \\
$6-8$ & $3.95 \pm 0.01$ & $4.10 \pm 0.01^{*}$ \\
$8-1$ & $3.76 \pm 0.01$ & $3.75 \pm 0.01$ \\
B5-b & & \\
Streptococcus thermophilus & $4.29 \pm 0.01$ & $4.29 \pm 0.01$ \\
$2-8$ & $4.45 \pm 0.01$ & $4.47 \pm 0.01$ \\
501 & $4.46 \pm 0.01$ & $4.47 \pm 0.01$ \\
510 &
\end{tabular}

${ }^{1}$ Lactobacillus and Streptococcus strains were grown in $10 \%$ skim milk with $0.5 \%$ D-allulose (allulose) or without (control) at $37^{\circ} \mathrm{C}$ for $1 \mathrm{~d}$.

${ }^{2}$ Means \pm SE from 2 culture samples.

*Values significantly different from control $(P<0.05)$.

where $A_{\text {blank1 }}$ was the absorbance of the control (without ACE inhibition), $\mathrm{A}_{\text {blank2 }}$ was the absorbance of the reagent blank, and $\mathrm{A}_{\text {sample }}$ was the absorbance of the sample. Absorbance was measured at a wavelength of $450 \mathrm{~nm}$.

\section{SOD Activity}

Stationary-phase bacterial cultures in TYG with and without D-allulose $(0.5 \%)$ were centrifuged at $1,800 \times$ $g$ for $15 \mathrm{~min}$; the pellets were washed twice with $0.85 \%$ $\mathrm{NaCl}$ and then mechanically disrupted with Fast Prep FP120 (ThermoSavant, Holbrook, NY) in $240 \mu \mathrm{L}$ of suspension buffer $\left(0.1 \mathrm{M} \mathrm{KH}_{2} \mathrm{PO}_{4}, \mathrm{pH} 7.0\right)$ and $100 \mu \mathrm{L}$ of lysis buffer $(0.3 \%$ SDS in $50 \mathrm{~m} M$ Tris- $\mathrm{HCl}, \mathrm{pH} 8.0)$. Cell debris was removed by centrifugation at $13,000 \times g$ for $10 \mathrm{~min}$, and the resulting supernatant was a cell-free extract. Superoxide-scavenging activity in the cell-free extract was determined by using a calorimetric SOD assay kit (Dojindo Molecular Tech., Kumamoto, Japan), in which a water-soluble tetrazolium salt, WST-1 (4-[3-(4-iodophenyl)-2-(4-nitrophenyl)-2 H-5-tetrazolio]1,3-benzene disulfonate sodium salt), is used (Ukeda et al., 1999). The superoxide anion generated by xanthine/xanthine oxidase (XO) reduces the WST-1 to water-soluble formazan, which exhibits absorbance at a wavelength of $450 \mathrm{~nm}$. The rate of reduction is linearly related to the XO activity, and is reduced by the presence of superoxide-scavenging substances. Superoxidescavenging activity determined with this method was estimated as SOD activity by using purified SOD from bovine erythrocytes (Sigma-Aldrich, St. Louis, MO) as the standard (Archibald and Fridovich, 1981). The enzyme reaction was performed at $37^{\circ} \mathrm{C}$. Protein concentrations in the cell-free extract were assayed using a BCA Protein Assay Kit (Pierce, Rockford, IL) with BSA as the standard.

\section{Statistical Analysis}

Results are expressed as means or means \pm standard error of 2 or 3 replicates analyzed using Student's $t$ test or the generalized linear model procedure of the SAS system (version 9.1; SAS Institute Inc., Cary, NC). Differences in multi-comparisons were evaluated by applying the Tukey's test or Dunnett's test. Statistical significance was defined as $P<0.05$.

\section{RESULTS}

\section{Effects of D-Allulose on Acid Production by Pure and Mixed Cultures of Yogurt Starter in Milk}

We examined the effects of adding $0.5 \%$ D-allulose on acid production in single yogurt starter cultures (Table 2 ). Addition of D-allulose significantly lowered acid production in the cultures of L. delbrueckii ssp. bulgaricus strains 6-8 and 8-1 relative to the control samples (i.e., without D-allulose). In the other cultures, we observed no significant decrease in acid production. Both strains 6-8 and 8-1 belong to L. delbrueckii ssp. bulgaricus. Strain 6-8 was selected for further study, because its suppression of acid production with D-allulose was higher than that of strain 8-1, although the difference was not significant (Table 2).

Next, we examined acid production by pairs of yogurt starter cultures (mixes of $L$. delbrueckii ssp. bulgaricus 6-8 or 5-10 and strains of $S$. thermophilus) grown in milk with $0.5 \%$ D-allulose (Table 3 ). The $\mathrm{pH}$ value in a mixed culture of L. delbrueckii ssp. bulgaricus 6-8 and S. thermophilus 501 was significantly higher with Dallulose than with control.

\section{Effects of D-Allulose on Acid Production by Probiotic Candidates in Milk}

We examined the effects of $0.5 \%$ D-allulose on the acid production of 4 Lactococcus strains in milk (Table 4). We observed a significant decrease in acid production with the addition of D-allulose to milk only in the case of strain $\mathrm{H} 61$.

\section{Acid Production by Selected Strains Grown with Different Sugars in Milk}

We selected strains 6-8, 501, H61, and ATCC $19257^{\mathrm{T}}$ for our analysis of sugar utilization. These strains were unable to grow in constituted medium with D-allulose as the only carbon source (data not shown). We exam- 
Table 3. $\mathrm{pH}$ values after fermentation of milk with or without D-allulose, ${ }^{1}$ to which mixed cultures of yogurt starters were added

\begin{tabular}{lcc}
\hline & \multicolumn{2}{c}{$\mathrm{pH}^{2}$} \\
\cline { 2 - 3 } Strain & Control & Allulose \\
\hline L. delbrueckii ssp. bulgaricus 6-8 with S. thermophilus & $3.66 \pm 0.01$ & $3.69 \pm 0.01$ \\
$2-8$ & $3.76 \pm 0.01$ & $3.85 \pm 0.01^{*}$ \\
501 & $3.63 \pm 0.01$ & $3.65 \pm 0.01$ \\
510 & & \\
L. delbrueckii ssp. bulgaricus 5-10 with S. thermophilus & $3.75 \pm 0.01$ & $3.74 \pm 0.01$ \\
$2-8$ & $3.69 \pm 0.01$ & $3.69 \pm 0.01$ \\
501 & $3.70 \pm 0.01$ & $3.69 \pm 0.01$ \\
\hline
\end{tabular}

${ }^{1}$ Lactobacillus delbrueckii ssp. bulgaricus 6-8 or 5-10 were grown with Streptococcus thermophilus strains in $10 \%$ skim milk with $0.5 \%$ D-allulose (allulose) or without (control) at $37^{\circ} \mathrm{C}$ for $1 \mathrm{~d} ; 1 \%$ inocula composed of 2 different strains (concentration of each inoculum was $0.5 \%$ ) were added to the milk.

${ }^{2}$ Means \pm SE from 2 culture samples.

*Values significantly different from control $(P<0.05)$

ined the effects of unassimilated sugar and sugar alcohol on acid production by the selected strains (Table 5). We used xylose and sorbitol for this experiment, because the test strains could not metabolize xylose and sorbitol (data now shown). The $\mathrm{pH}$ of the milk with Lactococcus strain H61 was not raised by adding xylose or sorbitol. In contrast, the $\mathrm{pH}$ of the milk with Lactococcus ATCC $19257^{\mathrm{T}}$ was lowered significantly by adding xylose or sorbitol. The $\mathrm{pH}$ of the milk with Lactobacillus strain 6-8 was increased significantly by adding both xylose and sorbitol, whereas the $\mathrm{pH}$ of the milk with Lactobacillus strain 5-10 was increased significantly only by xylose. Strain H61 was selected for further study to characterize the effect of D-allulose on the bacterial growth and probiotic activities of dairy lactic acid bacteria.

\section{Characterization of Effects of D-Allulose on Strain H61 Grown in Milk}

We next investigated dose response to D-allulose. We added D-allulose to milk to final concentrations of 0.1, $0.2,0.5$, or $1 \%$ (Table 6 ). Addition of D-allulose caused a dose-dependent increase in $\mathrm{pH}$ value.

Changes in milk $\mathrm{pH}$ value and viable cell count during cultivation of strain H61 cultures with or without D-allulose are shown in Figures 1a and 1b, respectively. We observed significant suppression of acid production by D-allulose at 8,24 , and $48 \mathrm{~h}$. For viable cell count, we observed an initial delay in the growth of strain H61 in D-allulose culture. However, the viable cell count of strain H61 in D-allulose culture markedly increased from 8 to $24 \mathrm{~h}$, and the count at $24 \mathrm{~h}$ in the D-allulose culture $(9.02 \pm 0.00 \log \mathrm{cfu} / \mathrm{mL})$ was comparable to the count at $24 \mathrm{~h}$ in the control culture $(9.04 \pm 0.06 \mathrm{log}$ $\mathrm{cfu} / \mathrm{mL} ; P=0.7400)$. At the end of the culture period $(48 \mathrm{~h})$, the viable count in the control culture $(8.91 \pm$
$0.02 \log \mathrm{cfu} / \mathrm{mL})$ tended to be lower than that in the D-allulose culture $(8.98 \pm 0.01 \log \mathrm{cfu} / \mathrm{mL} ; P=0.060)$. Analysis of D-allulose in milk revealed that D-allulose was not consumed by strain H61 (data not shown).

We also investigated the effects of fructose (0.5\%), an epimer of D-allulose, on acid production by strain H61 in milk after culture for $1 \mathrm{~d}$. Strain $\mathrm{H} 61$ can metabolize fructose (data not shown). The final $\mathrm{pH}$ values of the milk media were $4.31 \pm 0.00$ (control) and $4.22 \pm 0.00$ (fructose): these values were significantly different $(P$ $<0.001)$.

\section{Effects of D-Allulose on Bacterial Growth, Growth Efficiency, and Metabolite Production of Strain H61 Grown in Constituted Medium}

Changes in $\mathrm{pH}$ value and viable cell count of TYG culture during cultivation of strain H61 cultures with or without D-allulose are shown in Figures $2 \mathrm{a}$ and $2 \mathrm{~b}$, respectively. As in the case of milk culture, acid pro-

Table 4. $\mathrm{pH}$ values after fermentation of milk with or without D-allulose, ${ }^{1}$ to which probiotic candidate lactococci were added

\begin{tabular}{lcc}
\hline & \multicolumn{2}{c}{$\mathrm{pH}^{2}$} \\
\cline { 2 - 3 } Strain & Control & Allulose \\
\hline Lactococcus lactis ssp. cremoris & $4.21 \pm 0.01$ & $4.50 \pm 0.01^{*}$ \\
H61 & $4.28 \pm 0.01$ & $4.28 \pm 0.01$ \\
$7-1$ & $4.32 \pm 0.01$ & $4.31 \pm 0.01$ \\
ATCC 19257 & & \\
Lactococcus lactis ssp. lactis & $4.75 \pm 0.01$ & $4.75 \pm 0.01$ \\
N7 & & \\
\hline
\end{tabular}

${ }^{1}$ Lactococcus lactis $\mathrm{H} 61$, ATCC $19257^{\mathrm{T}}$, and $7-1$ were grown in $10 \%$ skim milk with $0.5 \%$ D-allulose (allulose) or without (control) at $30^{\circ} \mathrm{C}$ for 1 d. Lactococcus lactis N7 was grown in $10 \%$ skim milk with $0.5 \%$ D-allulose (allulose) or without (control) at $30^{\circ} \mathrm{C}$ for $2 \mathrm{~d}$.

${ }^{2}$ Means \pm SE from 2 culture samples.

*Values significantly different from control $(P<0.05)$. 
Table 5. Effects of different sugars on the $\mathrm{pH}$ values of milk to which selected strains were added ${ }^{1}$

\begin{tabular}{|c|c|c|c|}
\hline \multirow[b]{2}{*}{ Strain } & \multicolumn{3}{|c|}{$\mathrm{pH}^{2}$} \\
\hline & Control & Sorbitol & Xylose \\
\hline \multicolumn{4}{|l|}{ Lactococcus lactis ssp. cremoris } \\
\hline H61 & $4.20 \pm 0.01^{\mathrm{a}}$ & $4.20 \pm 0.01^{\mathrm{a}}$ & $4.19 \pm 0.01^{\mathrm{a}}$ \\
\hline ATCC $19257^{\mathrm{T}}$ & $4.32 \pm 0.01^{\mathrm{a}}$ & $4.28 \pm 0.01^{\mathrm{b}}$ & $4.28 \pm 0.01^{\mathrm{b}}$ \\
\hline \multicolumn{4}{|l|}{ Lactobacillus delbrueckii ssp. bulgaricus } \\
\hline $6-8$ & $3.99 \pm 0.01^{\mathrm{c}}$ & $4.04 \pm 0.01^{\mathrm{b}}$ & $4.11 \pm 0.01^{\mathrm{a}}$ \\
\hline $5-10$ & $4.08 \pm 0.01^{\mathrm{b}}$ & $4.08 \pm 0.01^{\mathrm{b}}$ & $4.13 \pm 0.01^{\mathrm{a}}$ \\
\hline
\end{tabular}

$\overline{{ }^{a-c} \text { Mean } \mathrm{pH} \text { values with different superscript letters within each treatment were significantly different }(P<}$ $0.05)$.

${ }^{1}$ Lactococcus and Lactobacillus were grown in $10 \%$ skim milk with $0.5 \%$ sorbitol (sorbitol) or $0.5 \%$ xylose (xylose), or without either (control), at $30^{\circ} \mathrm{C}$ (Lactococcus) or $37^{\circ} \mathrm{C}$ (Lactobacillus) for $1 \mathrm{~d}$.

${ }^{2}$ Means \pm SE from 2 culture samples.

duction was significantly suppressed by D-allulose for each cultivation time of 8,24 , and $48 \mathrm{~h}$. Despite the difference in acid production, viable cell count of strain $\mathrm{H} 61$ at 8 and $24 \mathrm{~h}$ was similar for the control and Dallulose cultures. At the end of the culture period (48 $\mathrm{h})$, the viable cell count in the control culture $(7.41 \pm$ $0.03 \log \mathrm{cfu} / \mathrm{mL}$ ) was significantly lower than that in the D-allulose culture $(7.93 \pm 0.05 \log \mathrm{cfu} / \mathrm{mL})$.

We calculated the molar growth $\mathrm{Y}_{\mathrm{G}}$ by dividing the dry weight of cells by the molar mass of consumed glucose. The $\mathrm{Y}_{\mathrm{G}}$ of cells for $1 \mathrm{~d}$ cultivation with D-allulose $(20.9 \pm 1.6)$ was comparable to the control $(22.1 \pm 0.6$; $P=0.5125)$.

We performed metabolite analyses of strain H61 with and without $0.5 \%$ D-allulose (Table 7 ). The main fermentation product of strain H61 cultured with or without D-allulose was lactate, with small amounts of acetate, formate, and ethanol; lactate production and glucose consumption were significantly lower in the presence of D-allulose.

\section{Probiotic Activities of Strain H61 Grown with D-Allulose}

We investigated the ACE-inhibitory capacity of strain H61 grown in milk with or without $0.5 \%$ D-allulose.

Table 6. Dose responses of D-allulose to $\mathrm{pH}$ values of milk fermented with strain $\mathrm{H} 61^{1}$

\begin{tabular}{lc}
\hline Allulose (\%) & $\mathrm{pH}^{2}$ \\
\hline 0 & $4.25 \pm 0.01^{\mathrm{a}}$ \\
0.1 & $4.28 \pm 0.01^{\mathrm{b}}$ \\
0.2 & $4.39 \pm 0.01^{\mathrm{c}}$ \\
0.5 & $4.60 \pm 0.01^{\mathrm{d}}$ \\
1.0 & $4.66 \pm 0.01^{\mathrm{e}}$ \\
\hline
\end{tabular}

${ }^{\mathrm{a} e} \mathrm{Mean} \mathrm{pH}$ values with different superscript letters were significantly different $(P<0.05)$.

${ }^{1}$ Lactocuccus lactis $\mathrm{H} 61$ was grown in $10 \%$ skim milk at $30^{\circ} \mathrm{C}$ for $1 \mathrm{~d}$.

${ }^{2}$ Means \pm SE from 2 culture samples. (a)

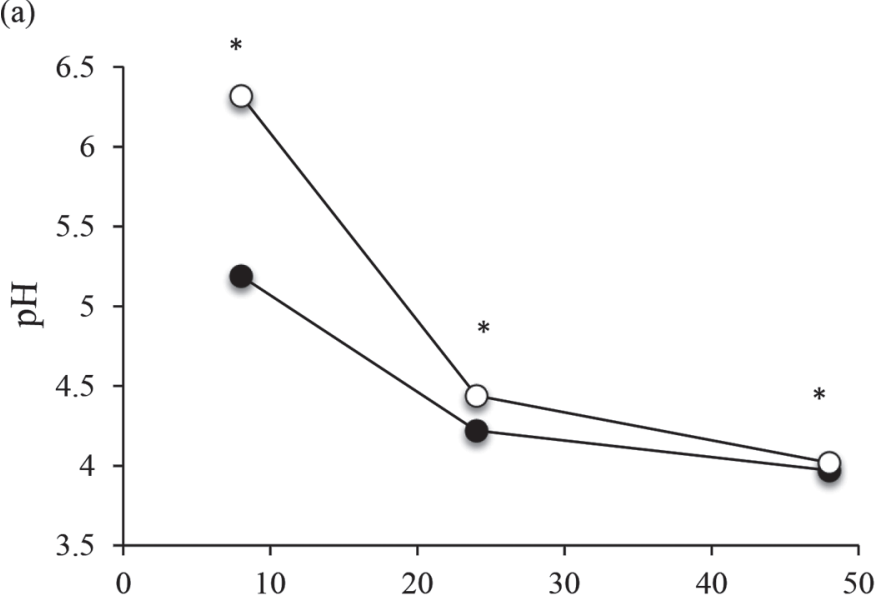

(b)

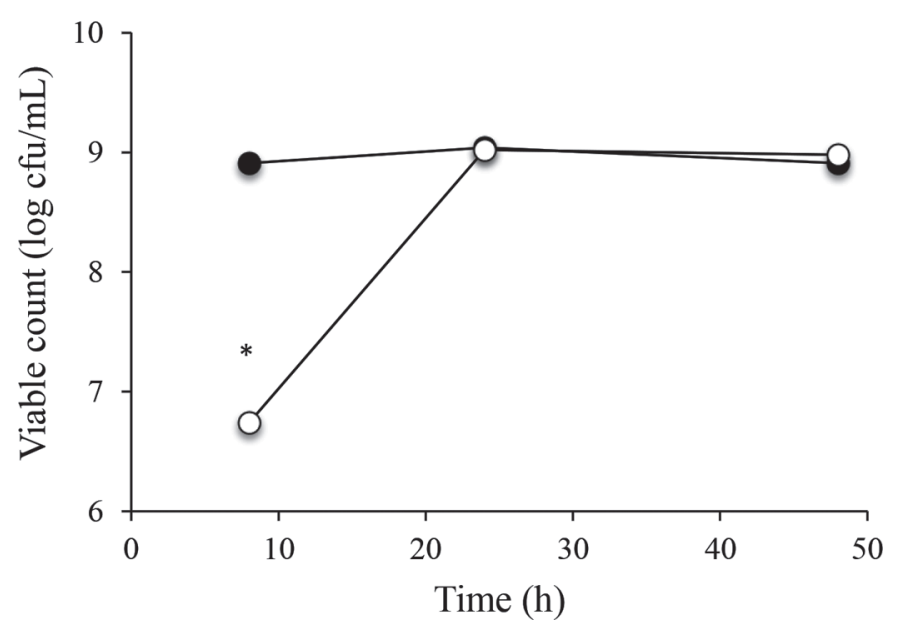

Figure 1. Time course of (a) $\mathrm{pH}$ value and (b) viable cell count in $10 \%$ skim milk with D-allulose (white circles) or without (control, black circles) and inoculated with Lactococcus lactis H61. Data are means from 2 to 3 culture samples. ${ }^{*} P<0.05$ indicates a significant difference between D-allulose culture and each control culture at each time. 
Table 7. Fermentation products from cultures of strain H61 grown with or without D-allulose ${ }^{1}$ in constituted medium

\begin{tabular}{|c|c|c|c|c|c|}
\hline \multirow[b]{2}{*}{ Group } & \multirow{2}{*}{$\begin{array}{l}\text { Glucose consumption } \\
\qquad(\mathrm{m} M)\end{array}$} & \multicolumn{4}{|c|}{ Metabolite production $(\mathrm{m} M)$} \\
\hline & & Lactate & Acetate & Formate & Ethanol \\
\hline Control & $19.4 \pm 0.3$ & $40.9 \pm 0.1$ & $0.7 \pm 0.0$ & $1.1 \pm 0.0$ & $0.3 \pm 0.1$ \\
\hline Allulose & $16.2 \pm 0.6^{* *}$ & $34.3 \pm 1.4^{* *}$ & $0.7 \pm 0.1$ & $1.1 \pm 0.1$ & $0.4 \pm 0.1$ \\
\hline
\end{tabular}

${ }^{1}$ Lactococcus lactis H61 was cultured in tryptone-yeast extract-glucose broth with $0.5 \%$ D-allulose (allulose) or without it (control) for $1 \mathrm{~d}$ at $30^{\circ} \mathrm{C}$. Results are means $\pm \mathrm{SE}$ from 3 culture samples.

$* *$ Values significantly different from control $(P<0.01)$.

The final $\mathrm{pH}$ values of the milk media were $4.17 \pm 0.01$ (control) and $4.32 \pm 0.00$ (D-allulose): these values were significantly different $(P<0.001)$. The ACE-inhibitory activity of strain $\mathrm{H} 61$ grown with D-allulose $(84.0 \% \pm$ $0.0 \%)$ was comparable the control $(84.2 \% \pm 0.1 \% ; P=$ $0.3739)$.

(a)

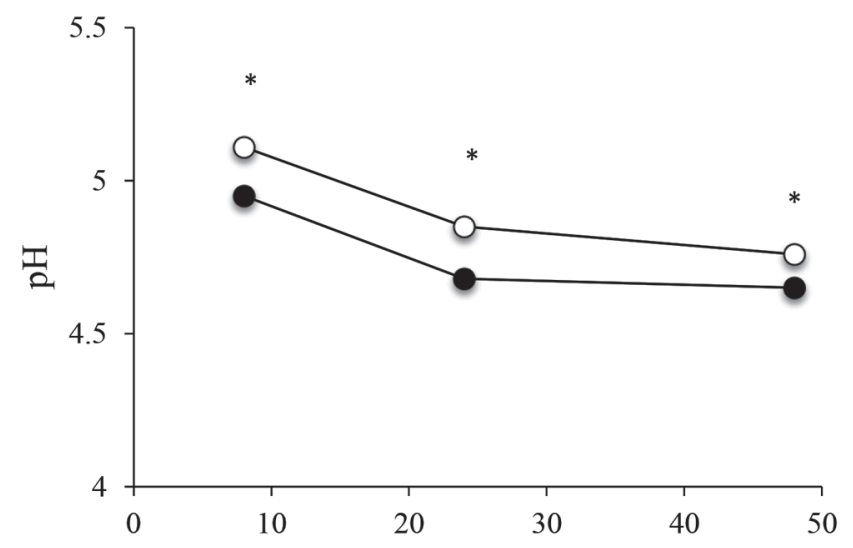

(b)

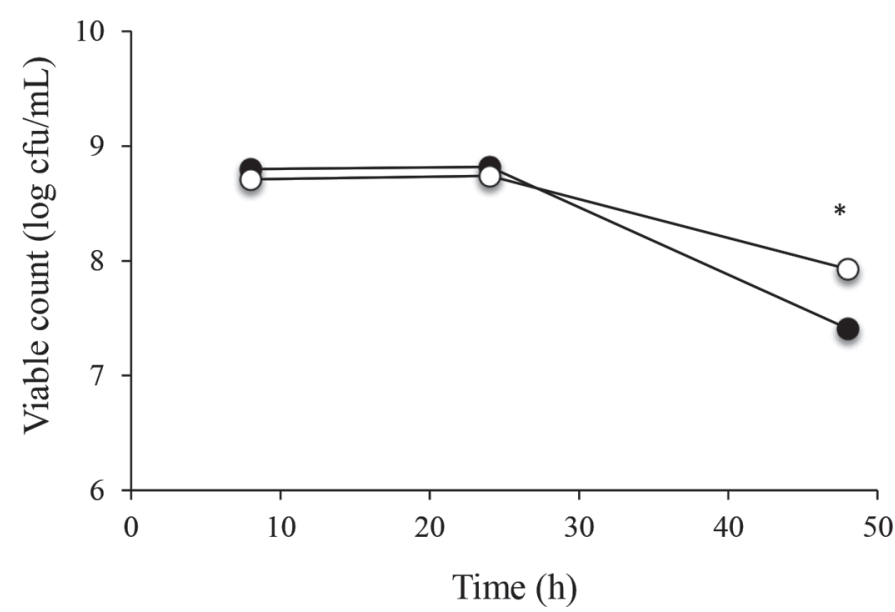

Figure 2. Time course of (a) $\mathrm{pH}$ value and (b) viable cell count in tryptone-yeast extract-glucose broth with D-allulose (white circles) or without (control, black circles), inoculated with Lactococcus lactis H61. Data are means of 3 culture samples. ${ }^{*} P<0.05$ indicates a significant difference between D-allulose culture and each control culture at each time.
We investigated the SOD activity of strain H61 grown with or without $0.5 \%$ D-allulose in TYG broth. The SOD activity of strain H61 grown with D-allulose (42.4 $\pm 3.9 \mathrm{U} / \mathrm{mg}$ of protein) was comparable to the control $(41.8 \pm 1.4 \mathrm{U} / \mathrm{mg}$ of protein; $P=0.8857)$.

\section{DISCUSSION}

In this study, we investigated the effects of the rare sugar D-allulose on the characteristics of dairy lactic acid bacteria and its application in the dairy product industry. The effect of D-allulose on acid production in milk was strain-specific. Acid production by certain yogurt starters (L. delbrueckii ssp. bulgaricus and $S$. thermophilus) was suppressed with D-allulose. We also observed suppression of acid production by $L$. delbrueckii ssp. bulgaricus (strains 6-8 and 5-10) with another sugar (xylose) and with a sugar alcohol (sorbitol). Among the dairy lactococci (which are used for manufacturing fermented milk and cheese), only $L$. lactis ssp. cremoris $\mathrm{H} 61$ showed reduced acid production in response to D-allulose in milk. Interestingly, acid production by strain $\mathrm{H} 61$ was not affected by fructose, xylose, or sorbitol. We therefore focused on strain H61 in our characterization of the effects of D-allulose for use in dairy product development.

Lactococcus lactis strains have a limited capacity to synthesize amino acids (Chopin, 1993) and depend on nitrogen sources (such as casein in milk) for growth. Therefore, proteolytic activity is critical to bacterial growth. We found that in strain H61, acid production was suppressed by D-allulose in TYG broth, which contains hydrolyzed casein, although its suppression effect was smaller than in milk during earlier fermentation. We assumed that bacterial proteolytic activity caused the observed suppression, in part, but what was the other D-allulose acid-suppression mechanism in milk and TYG broth? Park et al. (2007) reported the production of D-allose from D-allulose by 1 strain of Lactococcus lactis. In this study, we found that that strain H61 did not metabolize D-allulose, behaving differently from the L. lactis strain investigated by Park et al. (2007). The growth yield of cells generated per 
mole of glucose invested (the molar growth $\mathrm{Y}_{\mathrm{G}}$ ) is used widely to assay the molecular energetics of biochemical pathways (Loubiere et al., 1992). Because a generally accepted energetic yield value of 10.5 has been used as a biological constant (Bauchop and Elsden, 1960), the $\mathrm{Y}_{\mathrm{G}}$ of an organism that ferments glucose via the hexose diphosphate pathway should be $21 \mathrm{~g}$ (dry weight) of cells per mole of glucose. In this study, the $\mathrm{Y}_{\mathrm{G}}$ of strain $\mathrm{H} 61$ grown in the presence of D-allulose was comparable to that value. Low growth efficiency of bacteria can be caused by stressors such as low pH (Miyagi et al., 1994) or exposure to bile (Kimoto et al., 2002). However, the $\mathrm{Y}_{\mathrm{G}}$ value of strain H61 grown on TYG broth with Dallulose did not differ from the control. This finding indicated that energy production from glucose was not altered by adding D-allulose, and that strain H61 could not use D-allulose as an energy source in the presence of glucose: the energetic pathway was glycolysis alone. In fact, metabolism in strain $\mathrm{H} 61$ led to $>90 \%$ conversion of glucose to lactate, suggesting that the uptake of sugars by strain H61 is inhibited by D-allulose, reducing the acid production without changing the fermentation style. However, it is not clear why we observed suppression of acid production in milk in strain H61 but not in strain ATCC $19257^{\mathrm{T}}$, which belongs to the same subspecies. Strain ATCC $19257^{\mathrm{T}}$ did not metabolize D-allulose, and its fermentation style was not altered by D-allulose addition (data not shown). Comparison of strain H61 with ATCC $19257^{\mathrm{T}}$ can help identify the factors that affect acid production by D-allulose.

D-Allulose is present in common foods such as fruit juice $(21.5 \mathrm{mg} / 100 \mathrm{~g})$ and corn snacks $(47.0 \mathrm{mg} / 100 \mathrm{~g}$; Oshima et al., 2006). In addition, it is often produced by heating sucrose or fructose during cooking (Oshima et al., 2006). We found that, in the presence of D-allulose, beneficial properties such as the ACE-inhibitory activity of fermented milk and the SOD activity of cells grown in TYG broth were not affected in strain H61. A weak correlation has been reported between proteolytic enzyme activity and ACE-inhibitory activity in lactic acid bacteria (Fuglsang et al., 2003; RodríguezFigueroa et al., 2010). Thus, if bacterial proteolytic enzyme activity is affected by D-allulose, ACE-inhibitory activity may be altered. In addition, in our previous study (Kimoto-Nira et al., 2014), we found that the SOD activity of a certain strain of Lactococcus lactis could be altered by $\mathrm{C}$-source. In the present study, we showed that the ACE-inhibitory and SOD activities of strain $\mathrm{H} 61$ were not affected by D-allulose. In strains 7-1 and N7, these activities were not affected by Dallulose (data not shown). These are important results for the application of these strains as probiotic bacteria in D-allulose-containing medium.
D-Allulose may be useful for developing new probiotic dairy products from potential probiotic strain H61, because the acid production and growth suppression during earlier fermentation caused by D-allulose gradually decreases as milk fermentation progresses. Proteins glycated with D-allulose have high antioxidant activity in foods such as ice cream; they also have better emulsifying and foaming properties than proteins or proteins glycated with glucose (Puangmanee et al., 2008). We did not perform a rheological analysis of milk fermented with strain H61 and D-allulose; further study of this issue is needed.

\section{CONCLUSIONS}

The beneficial effects of D-allulose can be realized when D-allulose is not metabolized by lactic acid bacteria. The acid production of several dairy lactic acid bacteria in milk was affected by D-allulose, suggesting that this sugar could be used to regulate acid production in overfermented milk that would have a highly intense sour taste. D-Allulose is therefore likely to be useful in the manufacture of dairy products.

\section{ACKNOWLEDGMENTS}

We thank C. Koitabashi, X. Y. Yu, M. Nishimura, and $\mathrm{K}$. Yamasaki in our institute for their technical assistance.

\section{REFERENCES}

Archibald, F. S., and I. Fridovich. 1981. Manganese, superoxide dismutase, and oxygen tolerance in some lactic acid bacteria. J. Bacteriol. 146:928-936.

Bauchop, T., and S. R. Elsden. 1960. The growth of micro-organisms in relation to their energy supply. J. Gen. Microbiol. 23:457-469.

Chopin, A. 1993. Organization and regulation of genes for amino acids biosynthesis in lactic acid bacteria. FEMS Microbiol. Rev. $12: 21-37$.

Donkor, O. N., A. Henriksson, T. K. Singh, T. Vasiljevic, and N. P. Shah. 2007. ACE-inhibitory activity of probiotic yoghurt. Int. Dairy J. 17:1321-1331.

Fuglsang, A., F. P. Rattray, D. Niosson, and N. C. Nyborg. 2003. Lactic acid bacteria: Inhibition of angiotensin converting enzyme in vitro and in vivo. Antonie Van. Leeuwenhoek 83:27-34.

Han, W., A. Mercenier, A. Ait-Belgnaoui, S. Pavan, F. Lamine, I. I. van Sam, M. Kleerebrezem, C. Salvador-Cartier, M. Hisberues, L. Bueno, V. Theodorou, and J. Fioramonti. 2006. Improvement of an experimental colitis in rats by lactic acid bacteria producing superoxide dismutase. Inflamm. Bowel Dis. 12:1044-1052.

Hayashi, N., T. Iida, T. Yamada, K. Okuma, I. Takehara, T. Yamamoto, K. Yamada, and M. Tokuda. 2010. Study on the postprandial blood glucose suppression effect of d-psicose in borderline diabetes and the safety of long-term ingestion by normal human subjects. Biosci. Biotechnol. Biochem. 74:510-519.

Hébert, E. M., R. R. Raya, P. Tailliez, and G. S. de Giori. 2000. Characterization of natural isolates of Lactobacillus strains to be used as starter cultures in dairy fermentation. Int. J. Food Microbiol. 59:19-27. 
Hirota, T., K. Ohki, R. Kawagishi, Y. Kajimoto, S. Mizuno, Y. Nakamura, and M. Kitakaze. 2007. Casein hydrolysate containing the antihypertensive tripeptides Val-Pro-Pro and Ile-Pro-Pro improves vascular endothelial function independent of blood pressure-lowering effects: Contribution of the inhibitory action of angiotensinconverting enzyme. Hypertens. Res. 30:489-496.

Hishiike, T., M. Ogawa, S. Hayakawa, D. Nakajima, S. O'Charoen, H. Ooshima, and Y. Sun. 2013. Transepithelial transports of rare sugar D-psicose in human intestine. J. Agric. Food Chem. 61:73817386.

Iida, T., Y. Kishimoto, Y. Yoshikawa, N. Hayashi, K. Okuma, M. Tohi, K. Yagi, T. Matsuo, and K. Izumori. 2008. Acute D-psicose administration decreases the glycemic responses to an oral maltodextrin tolerance test in normal adults. J. Nutr. Sci. Vitaminol. (Tokyo) 54:511-514.

Kimoto, H., S. Ohmomo, and T. Okamoto. 2002. Cholesterol removal from media by lactococci. J. Dairy Sci. 85:3182-3188.

Kimoto-Nira, H., R. Aoki, K. Mizumachi, K. Sasaki, H. Naito, T. Sawada, and C. Suzuki. 2012a. Interaction between Lactococcus lactis and Lactococcus raffinolactis during growth in milk: Development of a new starter culture. J. Dairy Sci. 95:2176-2185.

Kimoto-Nira, H., R. Aoki, K. Sasaki, C. Suzuki, and K. Mizumachi. 2012b. Oral intake of heat-killed cells of Lactococcus lactis strain H61 promotes skin health in women. J. Nutr. Sci. 1:e18.

Kimoto-Nira, H., N. Moriya, H. Ohmori, and C. Suzuki. 2014. Altered superoxide dismutase activity by carbohydrate utilization in a $\mathrm{Lac}$ tococcus lactis strain. J. Food Prot. 77:1161-1167.

Kimoto-Nira, H., S. Yamasaki, K. Sasaki, N. Moriya, A. Takenaka, and C. Suzuki. 2015. New lactic acid bacterial strains from traditional Mongolian fermented milk products have altered adhesion to porcine gastric mucin depending on the carbon source. Anim. Sci. J. 86:325-332.

LeBlanc, J. G., S. del Carmen, A. Miyoshi, V. Azevedo, F. Sesma, P. Langella, L. G. Bermudez-Humaran, L. Warrerlot, G. Perdigon, and A. de Moreno de LeBlanc. 2011. Use of superoxide dismutase and catalase producing lactic acid bacteria in TNBS induced Crohn's disease in mice. J. Biotechnol. 151:287-293.

Lee, W.-K., H.-J. Lim, S.-Y. Kim, H. Kimoto, S. Ohmomo, Y. Tashiro, and H. Takebe. 2005. Hypocholesterolemic effect of Lactococcus lactis ssp. lactis biovar diacetylactis N7 and Lactococcus lactis ssp. lactis 527 strains in SD rats. Biosci. Microflora 24:11-16.

Loubiere, P., P. Salou, M. J. Leroy, N. D. Lindley, and A. Pareilleux. 1992. Electrogenic malate uptake and improved growth energetics of the malolactic bacterium Leuconostoc oenos grown on glucosemalate mixtures. J. Bacteriol. 174:5302-5308.
Matsuo, T., Y. Baba, M. Hashiguchi, K. Takeshita, K. Izumori, and H. Suzuki. 2001. Dietary D-psicose, a C-3 epimer of D-fructose, suppresses the activity of hepatic lipogenic enzymes in rats. Asia Pac. J. Clin. Nutr. 10:233-237.

Miyagi, A., H. Ohta, T. Kodama, K. Fukui, K. Kato, and T. Shimono. 1994. Metabolic and energetic aspects of the growth response of Streptococcus rattus to environmental acidification in anaerobic continuous culture. Microbiology 140:1945-1952

Mu, W., W. Zhang, Y. Feng, B. Jiang, and L. Zhou. 2012. Recent advances on applications and biotechnological production of Dpsicose. Appl. Microbiol. Biotechnol. 94:1461-1467.

Nakamura, T., J. Mizutani, K. Yamada, N. Yamamoto, M. Takeshi, and K. Takazawa. 2011. Casein hydrolysate containing Val-ProPro and Ile-Pro-Pro improves central blood pressure and arterial stiffness in hypertensive subjects: A randomized, double-blind, placebo-controlled trial. Atherosclerosis 219:298-303.

Oshima, H., I. Kimura, and K. Izumori. 2006. Psicose contents in various food products and its origin. Food Sci. Technol. Res. 12:137143.

Park, H. Y., C. S. Park, H. J. Kim, and D. K. Oh. 2007. Substrate specificity of a galactose 6-phosphate isomerase from Lactococcus lactis that produces d-allose from d-psicose. J. Biotechnol. 132:8895.

Puangmanee, S., S. Hayakawa, Y. Sun, and M. Ogawa. 2008. Application of whey protein isolate glycated with rare sugars to ice cream. Food Sci. Technol. Res. 14:457-466.

Rodríguez-Figueroa, J. C., R. Reyes-Diaz, A. F. Gonzalez-Cordova, R. Troncoso-Rojas, I. Vargas-Arispuro, and B. Vallejo-Cordoba. 2010. Angiotensin-converting enzyme inhibitory activity of milk fermented by wild and industrial Lactococcus lactis strains. J. Dairy Sci. 93:5032-5038.

Takeshita, K., A. Suga, G. Takada, and K. Izumori. 2000. Mass production of D-psicose from d-fructose by a continuous bioreactor system using immobilized D-tagatose 3 -epimerase. J. Biosci. Bioeng. 90:453-455.

Thompson, J. L., K. Lopetchrat, and M. A. Drake. 2007. Preferences for commercial strawberry drinkable yogurts among African American, Caucasian, and Hispanic consumers in the United States. J. Dairy Sci. 90:4974-4987.

Ukeda, H., D. Kawana, S. Maeda, and M. Sawamura. 1999. Spectrophotometric assay for superoxide dismutase based on the reduction of highly water-soluble tetrazolium salts by xanthine-xanthine oxidase. Biosci. Biotechnol. Biochem. 63:485-488. 